remain exactly alike as compared with one another. With a further decrease in the force of the impulse, there comes a moment when one or another impulse ceases to resemble the others and appears shorter. This moment is defined as the 'beginning of fluctuation'. When the force of the stimulus is still further decreased, there is a new moment when one or the other impulses vanishes completely-'middle of fluctuation'. 'The next moment, when all of the impulses vanish, is considered the individual threshold of sensitivity of the given observer, $i$.

Numerous measurements have shown that the beginning of fluctuation comes when the intensity of the stimulus is four times the individual threshold $(6 \mathrm{db}$.$) . and the middle of fluctuation comes when the$ intensity is 1.6 times the threshold $(2 \mathrm{db}$.). The intensity of 0.6 of the threshold $(0.6 i)$ represents the amplitude of fluctuation, and one and the same values were obtained for hearing, vision and touch. The same value of 0.6 of the threshold was obtained for hearing within the limits of $200-3,000$ Herz, although the sensitivity of the ear changes 1,000 times within this range.

The phenomenon of fluctuation of the threshold of sensitivity can be explained if we realize that our sensations increase in jumps, or steps, and, furthermore, that the size of each such step is equivalent to the amplitude of fluctuation, that is, to $0.6 i$ (where $i$ is the threshold of sensitivity). I find five such steps, after which they become unobservable as compared to the increased loudness. (Fig. 1).

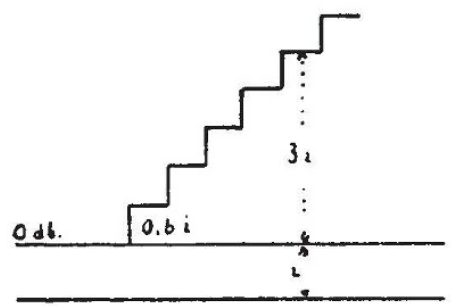

Fig. 1 .

Another series of experiments which confirms these results consisted of the following :

A number of impulses having a duration of $0.75 \mathrm{sec}$. were given, one after the other, at intervals of $0.75 \mathrm{sec}$. Further, two impulses were given simultaneously, one for vision (a neon lamp connected to a case having an opening of $0.5 \mathrm{~cm}$.), and the other for hearing (a telephone). Thus both impulses are received at the same time. The visual impulse serves as an indicator and its brightness remains constant throughout the duration of the experiments; the loudness of the sound impulse changes. At the beginning, both impulses are of the same duration-.-both begin and end simultaneously (Fig. 2a). As the loudness is decreased, the sound impulse begins to lag, at first scarcely noticeably (Fig. $2 b$ ); later it lags by half the visual impulse (Fig. $2 c$ ), and at the end there remains but one element of sensation, one sound dot at the end of the visual impulse (Fig. 2d). This takes place when the intensity of sound is equal to $1 \cdot 6 i$ (when the loudness is $2 \mathrm{db}$.), that is, exactly at an intensity which corresponds to the middle of fluctuation.

The time by which the sound impulse lags corresponds exactly to the time necessary for the accumu-

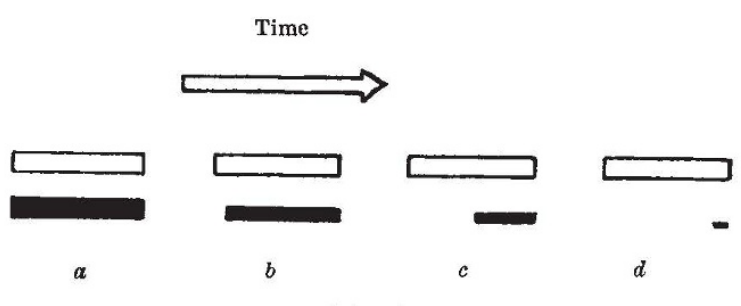

Fig. 2.

lation of the elementary work of sound $(1 \cdot 6 i t)$, which is necessary for one element of sensation.

The experiments confirmed very well the equation connecting the intensity of sound, $I$, the individual threshold of the observer, $i$, the duration of the impulse, $t$, and the extent of the lag $\Delta t$ :

$$
10 \lg \frac{I}{i}=2+10 \lg \frac{t}{\Delta t}
$$

The visual and sound impulses can be interchanged : the sound will then serve as an indicator and the visual impulse will vary in brightness. The visual impulse will then lag and will be shortened, as shown in Fig. 2. The element of visual sensation will be obtained when the intensity of light is equal to $1 \cdot 6 i$, and the time of the lag can be determined from equation (1). Experiment has shown that the same thing occurs with the tactual impulse. In this case, the indicator may be either the visual or the sound impulse.

Other experiments with impulses of greater duration have shown that the time for the accumulation of the elementary work of the impulse may reach many seconds.

Institute of Architecture,

Samuel Lifshitz. Moscow.

${ }^{1}$ Lifshitz, S., J. Acous. Soc. Amer., 11 (1.938).

${ }^{2}$ Lifshitz, S., J. Acous. Soc. Amer., 5, 31 (1933) ; 7, 213 (1936)

\section{Catalysis by Activated Copper Sulphide}

$60 \mathrm{gm}$. carefully purified acetaldehyde and $30 \mathrm{gm}$. copper sulphide, activated by oxidizing a part of its surface into cupric sulphate, were sealed in a tube of Pyrex glass and kept at $15-20^{\circ}$ for 24 days. At the end of this time, 60 per cent of the reactant was converted into paraldehyde, which was identified by determining the boiling point, the melting point and the mixed melting point with an authentic sample. In a control experiment without catalyst, 98 per cent of the acetaldehyde could be recovered.

It follows that the activated copper sulphide is a catalyst not only for the conversion of certain petroleum hydrocarbons ${ }^{1}$ but also for a low molecular polymerization involving the opening of a carbonyl bond.

\section{Albert WassermanN.} 1 Wassermann, Brit. Pat., 499958, French Pat., 838379 , U.S.A. Pat., Trans. Farad. Soc., 35, 1022 (1939). 